\title{
Recomendaciones para la valoración nutricional del paciente crítico
}

\author{
Juan Carlos Montejo G onzález ${ }^{1}$, Jesús M Culebras-Fernández ${ }^{2}$, \\ Abelardo García de Lorenzo y Mateos ${ }^{3}$.
}

\section{Recommendations for the nutritional assessment of critically ill patients}

Undernutrition affects 30 to $50 \%$ of hospitalized patients. The identification of patients with undernutrition or in risk to become undernourished is of utmost importance to prescribe a timely nutritional support. This article performs a critical review of anthropometrical, biochemical and functional parameters used for nutritional assessment. Prognostic indexes and other parameters used for the assessment of nutritional risk are also discussed. Some tools are proposed for the diagnosis of hospital malnutrition (Rev Méd Chile 2006; 134: 1051-8).

(Key words: Critical illness; Malnutrition; Nutrition assessment)

\begin{abstract}
Recibido el 19 de octubre, 2005. Aceptado el 10 de mayo, 2006.
${ }^{1}$ Servicio de Cuidados Intensivos, Hospital Universitario 12 de Octubre, Madrid. ${ }^{2}$ Servicio de Cirugía, Complejo Asistencial de León, León. ${ }^{3}$ Fundación Abbott y Universidad Autónoma de Madrid, España.
\end{abstract}

L a desnutrición continúa siendo la causa más frecuente de aumento de la morbimortalidad y uno de los principales problemas de salud en todo el mundo, afectando de forma muy especial a un colectivo concreto, como es el de los pacientes hospitalizados, donde la incapacidad de ingesta y la enfermedad son comunes, tomando entidad propia bajo la denominación de desnutrición hospitalaria ${ }^{1}$. La desnutrición afecta a 30\%$50 \%$ de los pacientes hospitalizados de todas las edades, tanto por causas quirúrgicas como médicas, aumentando a medida que se prolonga la hospitalización ${ }^{2}$.

Correspondencia a: Juan Carlos Montejo González. Cuidados Intensivos, Hospital Universitario 12 de Octubre, Madrid, España. E mail: jmntejo @telefonica.net
Merece destacarse que, desde los primeros estudios publicados sobre prevalencia de desnutrición hospitalaria, hasta los estudios más recientes, los porcentajes de pacientes hospitalizados desnutridos no han variado sustancialmente. Como causas de esta desnutrición cabe destacar la enfermedad, los procedimientos diagnósticos y terapéuticos y el escaso énfasis concedido al estado nutricional en la historia clínica $\mathrm{y}$, por ende, en los sistemas de codificación, en muchos casos debido al desconocimiento generalizado que existe sobre este problema, con el consiguiente fallo en la detección y empeoramiento de la desnutrición durante la estancia hospitalaria.

Es importante identificar a los pacientes desnutridos, o con riesgo de desnutrición, a fin de instaurar, lo antes posible, un soporte nutricional adecuado ${ }^{3}$. Estaríamos claramente en situación de mala práctica médica si hacemos caso omiso de las recomendacio- 
nes de nutrición artificial en el paciente crítico ${ }^{4}$, en la insuficiencia renal aguda ${ }^{5}$, en la insuficiencia hepáti$\mathrm{ca}^{6}$, en la pancreatitis aguda ${ }^{7}$, en la insuficiencia respiratoria ${ }^{8}$, en el síndrome de intestino corto $^{9}$, en la diabetes mellitus ${ }^{10}$, en los cánceres de aparato digestivo ${ }^{11}$, en el paciente con SIDA $^{12}$, en el gran quemado ${ }^{13}$, en los pacientes politraumatizados ${ }^{14}$, en la sepsis ${ }^{15}$, o después de un trasplante de células precursoras hematopoyéticas ${ }^{16}$.

Para ello son necesarios sistemas informatizados de detección o tamizaje de población (tanto al ingreso como durante la estancia hospitalaria) que actúen como alarmas. Estas herramientas deben de ser aplicables en la mayoría de los hospitales, $\mathrm{y}$ tener capacidad de aportar datos reproductibles, significativos y fiables ${ }^{17}$, para predecir los resultados de otros métodos más sofisticados.

Existen diferentes parámetros destinados a la valoración del estado nutricional. Estos parámetros pueden ser de utilidad para valorar el estado nutricional previo al ingreso de los pacientes. No obstante, su aplicación en los pacientes críticos es problemática, debido a que la interpretación de los resultados se encuentra interferida por los cambios originados por la enfermedad aguda y por las medidas de tratamiento ${ }^{18}$. Esto es particularmente cierto en lo que se refiere a las variables antropométricas, que se encuentran muy afectadas por las alteraciones en la distribución hídrica de los pacientes críticos. Los marcadores bioquímicos (índice creatinina/talla, albúmina sérica, etc.) se encuentran también interferidos como consecuencia de los cambios metabólicos, que modifican los procesos de síntesis y degradación. Las proteínas de vida media corta (prealbúmina, proteína ligada al retinol) no son indicativas de estado nutricional aunque sí informan sobre la adecuada respuesta al aporte de nutrientes y sobre la concurrencia de nuevas situaciones de estrés metabólico. Los parámetros de estimación funcional, como los tests de función muscular, son también difíciles de aplicar en un gran número de pacientes críticos, debido al tratamiento con fámacos que afectan a la función muscular 0 a la presencia de polineuropatía. Todo ello indica que no existen parámetros fiables para la valoración del estado nutricional en estos pacientes.

Otros métodos, teóricamente más precisos, como la impedancia bioeléctrica, requieren ser más estudiados en estos pacientes antes de poder ser recomendados.

\section{EVALUACIÓN DE LA MALNUTRICIÓN EN EL PACIENTE CRÍTICO}

En el paciente crítico, la malnutrición puede ser preexistente, manifestarse al ingreso o desarrollarse de forma evolutiva, favorecida por el estado hipercatabólico e hipermetabólico. La prevalencia de malnutrición oscila entre $30 \%$ y $60 \%$ de los enfermos hospitalizados, siendo aún más elevada en el paciente grave ${ }^{19}$, debido a la alteración en el metabolismo de los diferentes sustratos y al déficit de nutrientes ${ }^{20}$.

La valoración nutricional en el paciente crítico tiene como objetivos teóricos evaluar, de forma específica, el riesgo de mortalidad y morbilidad de la malnutrición, identificar y separar de forma individualizada las causas y consecuencias de la malnutrición y analizar el grupo de enfermos con mayor posibilidad de beneficiarse del soporte nutricional.

Para la valoración del estado nutricional en el paciente crítico se recurre normalmente a la utilización de los métodos habitualmente empleados en otros pacientes (variables antropométricas, marcadores bioquímicos y pruebas funcionales).

\section{VARIABLES ANTROPOMÉTRICAS}

Las variables antropométricas evalúan y detectan la malnutrición preexistente al ingreso del paciente crítico. No obstante, los cambios corporales y la evolución del estado de hidratación en estos pacientes, invalidan a este grupo de variables como parámetros de seguimiento nutricional y de pronóstico en los pacientes críticos.

Peso. Mide de forma simplificada el total de los componentes corporales. Una pérdida involuntaria de peso superior a $10 \%$ en los últimos seis meses o el rápido desarrollo de la misma, son signos clásicos de malnutrición, cuyo valor no ha sido analizado en los pacientes críticos.

Indice de masa corporal (IMC). Evalúa la relación entre el peso y la talla. Si bien se considera como rango normal un IMC comprendido entre $18,5 \mathrm{y}$ $24,9 \mathrm{~kg} / \mathrm{m}^{2}$, índices inferiores a $20 \mathrm{~kg} / \mathrm{m}^{2}$ son indicativos de malnutrición y se asocian con un aumento significativo en la mortalidad en diferentes tipos de pacientes ${ }^{21,22}$. En caso de no ser 
posible pesar, tallar o conocer la pérdida de peso, se recurrirá a otras determinaciones de segmentos corporales: circunferencia muscular del brazo (CMB), índice talón-rodilla (ITR), longitud del cúbito. En caso de que todo ello sea imposible, el IMC puede estimarse a través del aspecto del enfermo: normal, sobrepeso, caquexia.

Otras variables antropométricas. Las más utilizadas son: el pliegue del tríceps y el área muscular del brazo. Ambos métodos tienen escasa utilidad en la valoración nutricional de los pacientes críticos.

Valoración subjetiva global ${ }^{23}$. Es un método propuesto por Detsky y cols ${ }^{24}$ para valorar el estado nutricional en pacientes que van a ser sometidos a cirugía digestiva y en pacientes oncológicos. Está basada en la aplicación de dos grupos de datos: la historia clínica y la historia dietética, por un lado, y la exploración física (prestando especial atención al estado de la masa muscular y la masa grasa del individuo), por otro. Mediante la consideración de ambos factores el explorador puede clasificar, de manera subjetiva, el estado nutricional del paciente como bien nutrido, moderadamente desnutrido o en situación de desnutrición grave. La valoración global subjetiva, a pesar de su aparente sencillez, requiere un cierto grado de experiencia en su realización. No existen experiencias que comuniquen los resultados de la aplicación de este método a los pacientes críticos.

\section{VARIABLES BIOQUÍMICAS}

Al igual que ocurre con los parámetros antropométricos, las variables bioquímicas se encuentran interferidas por los cambios que tienen lugar en los pacientes críticos, por lo que su interés en la interpretación del estado nutricional es limitado.

Variables bioquímicas indicativas del estado de las proteínas musculares

Índice creatinina/talla. Es un indicador de la cantidad de masa muscular, dado que la creatinina es un producto final de la degradación de la creatina, que se encuentra principalmente en el tejido muscular. La creatinina es excretada por orina sin sufrir otros cambios metabólicos: por ello, la magnitud de la eliminación de creatinina muestra el grado de catabolismo muscular y dependerá de la cantidad de masa muscular. En ausencia de insuficiencia renal, la correlación de la eliminación diaria de creatinina con la talla del paciente (índice creatinina/altura) está en relación con la cantidad de masa muscular y el valor puede compararse con los considerados normales, de acuerdo a tablas previamente establecidas para la población normal. La principal limitación de esta técnica es, aparte de la insuficiencia renal, que invalida el resultado, la necesidad de recoger la orina de $24 \mathrm{~h}$ durante un periodo de 3 días consecutivos, con el fin de hacer la media diaria de eliminación. Además, el grado de función hepática, diversos tratamientos (como los diuréticos), la cantidad de aporte proteico y la situación de estrés metabólico inducida por la enfermedad grave, modifican de manera importante la utilidad de esta técnica. En ausencia de estas limitaciones, un índice creatinina/altura entre $60 \%$ y $80 \%$ del valor de referencia supondría una depleción proteica moderada, en tanto que cifras inferiores a $60 \%$ deberían ser calificadas como depleción proteica grave. En el paciente crítico el índice creatinina/talla puede detectar la malnutrición al ingreso, pero carece de valor pronóstico o de seguimiento de forma aislada ${ }^{25}$.

3-Metil-histidina. Es un aminoácido derivado del metabolismo muscular proteico. Sus valores aumentan en situaciones de hipercatabolismo y disminuyen en ancianos y en pacientes desnutridos. En el paciente crítico es un parámetro de seguimiento nutricional, renutrición y catabolismo muscular.

Excreción nitrogenada. Es un método habitual de medición del catabolismo proteico. Para su cuantificación se determina el nitrógeno ureico en orina de $24 \mathrm{~h}$ (equivalente a la urea multiplicada por 0,56$)$ y se añade una cantidad correspondiente a la estimación de las pérdidas nitrogenadas no urinarias (habitualmente, 2-3 gr/día). Sus valores presentan también variaciones en relación con el volumen intravascular, el aporte nitrogenado y la función renal. En el paciente crítico es un índice de la intensidad de la respuesta metabólica al estrés, considerándose estrés leve si la eliminación nitrogenada es de 5-10 gr/día, estrés moderado en caso de valores de 10-15 gr/día y estrés grave si la pérdida nitrogenada diaria es superior a $15 \mathrm{gr}$. 
Balance nitrogenado. Es un buen parámetro de renutrición en pacientes posoperados con estrés o desnutrición moderada. En el paciente crítico no es válido como parámetro de desnutrición y seguimiento nutricional, pero sí como índice de pronóstico nutricional. Debido a la elevada eliminación de nitrógeno presente, no es posible conseguir balances nitrogenados positivos en las fases iniciales de la enfermedad grave, por lo que, a pesar del soporte nutricional, los pacientes presentan persistentemente balance nitrogenado negativo durante los primeros días. En la fase de recuperación es cuando podrá apreciarse balance nitrogenado positivo si el soporte nutricional es adecuado a la situación del paciente.

Variables bioquímicas indicativas del estado de las proteínas viscerales

Albúmina. Es el parámetro bioquímico más frecuentemente utilizado en la valoración nutricional. Los valores de albúmina al ingreso tienen valor pronóstico: valores inferiores al límite nor$\mathrm{mal}(3,5 \mathrm{~g} / \mathrm{dl})$ se asocian con un incremento en la morbilidad y la mortalidad de los pacientes. No obstante, dichos valores son poco sensibles a los cambios agudos ${ }^{26}$ del estado nutricional (por la elevada vida media de la albúmina: 20 días). La albúmina sérica tampoco es un buen parámetro de seguimiento nutricional, aunque sus valores pueden relacionarse con la extensión de la lesión ${ }^{27}$

Prealbúmina. Su vida media corta (2 días), la convierte en un parámetro de evolución y seguimiento en el paciente crítico ${ }^{28}$, habiéndose apreciado que la prealbúmina es el parámetro más sensible a los cambios en el estado nutricional. No obstante, sus valores están interferidos por factores no relacionados con el estado nutricional: disminuyen en la infección y en la insuficiencia hepática y aumentan en el falla renal.

Proteína ligada al retinol. Su vida media corta (12 h) la convierte también en un marcador de seguimiento nutricional. Sus niveles aumentan con la ingesta de vitamina $\mathrm{A}$, disminuyen en la enfermedad hepática, infección y estrés grave. Carece de valor en pacientes en insuficiencia renal.

Transferrina. Es una beta-globulina de síntesis fundamentalmente hepática, cuya función principal es la del transporte de hierro en el plasma. Presenta una baja sensibilidad y especificidad cuando se analiza de forma individual. Sus niveles plasmáticos están aumentados en la anemia ferropénica ${ }^{29}$ y disminuidos en la enfermedad hepática, sepsis, síndrome de malabsorción y alteraciones inespecíficas inflamatorias ${ }^{30}$.

El déficit crónico de hierro, la politransfusión y las alteraciones en la absorción intestinal, lo invalidan como parámetro nutricional en el paciente crítico (Su vida media es de 8-10 días).

Somatomedina. Se trata de un péptido de bajo peso molecular, cuya síntesis está regulada por la hormona de crecimiento y el factor I de la insulina. Mide la intensidad de la respuesta metabólica de la agresión y es un buen parámetro de seguimiento nutricional. La complejidad en su determinación y su elevado costo limitan su uso.

Otras proteínas. La proteína C, alfa-1-antitripsina, alfa-1-glicoproteina, alfa-2-macroglobulina, la fibronectina, el fibrinógeno y la haptoglobina, son proteínas inespecíficas, cuyo valor puede encontrarse relacionado con la intensidad de la respuesta metabólica. No obstante, pueden alterarse también por un gran número de situaciones no relacionadas con el estado nutricional.

Colesterol. Los niveles de colesterol sérico se consideran como un parámetro de valoración nutricional ${ }^{31,32}$. Un nivel bajo de colesterol sérico ha sido observado en pacientes desnutridos, con insuficiencia renal, hepática y síndrome del malabsorción. La presencia de hipocolesterolemia puede ser indicativa de malnutrición en los pacientes críticos y se relaciona con un incremento en la mortalidad.

\section{Parámetros de estimación funcional}

Parámetros de función muscular. El análisis de la fuerza muscular, tanto de forma activa (fuerza de la musculatura respiratoria, capacidad de aprehensión), como pasiva (respuesta de contracción y relajación muscular a diferentes intensidades eléctricas) han sido utilizados como indicadores del estado nutricional. Sus valores fueron más sensibles y específicos en la predicción de complicaciones quirúrgicas que marcadores bioquímicos como la albúmina o la transferrina. No obstante, en el paciente crítico los test de función muscular pueden estar alterados por factores muy diversos 
como el uso de sedo-analgesia o la existencia de polineuropatías.

Parámetros de función inmunológica. La disminución en el recuento total de linfocitos $(<1.500)$, el índice de CD3/CD4 $(<50)$ y la ausencia en la respuesta de inmunidad celular retardada, se han relacionado con la malnutrición. En el paciente crítico, tanto los recuentos linfocitarios como los test de función inmunitaria pueden estar alterados por un gran número de situaciones clínicas o por la administración de medicamentos. Estos parámetros pueden tener valor en el seguimiento evolutivo de enfermos críticos que muestran déficit en la inmunidad al ingreso.

\section{ÍNDICES PRONÓSTICOS NUTRICIONALES}

Están dirigidos a la predicción del riesgo quirúrgico, el desarrollo de complicaciones posoperatorias y la indicación del soporte nutricional en función de la valoración del estado nutricional al ingreso de los pacientes. El más utilizado es el índice de Bristian ${ }^{27}$, validado para pacientes quirúrgicos pero no para pacientes críticos.

\section{OTROS PARÁMETROS UTIUZABLES}

PARA LA VALORACIÓN NUTRICIONAL EN PACIENTES CRÍTICOS

El análisis de la activación con neutrones, que mide el cálculo del nitrógeno corporal total, la impedancia bioeléctrica, que evalúa el volumen corporal total de agua y los isótopos de potasio, que calculan la masa tisular total magra, son técnicas aún experimentales, de escasa utilidad en el paciente crítico en el momento actual. Entre las técnicas citadas, la impedancia bioeléctrica es la que cuenta con mayor potencialidad de desarrollo para su aplicación a pacientes críticos; su facilidad de empleo la convierte en una técnica accesible «a pie de cama», aunque es preciso, antes de que su empleo sea generalizado, definir previamente los parámetros de normalidad» de la técnica en estos pacientes y validar la implicación de los cambios apreciados respecto a poblaciones normales ${ }^{33,34}$.

\section{RECOMENDACIONES}

No hay estudios sobre la utilidad de los parámetros antropométricos o de los marcadores bioquímicos más frecuentes en la valoración nutricional de los pacientes críticos, por lo que no puede recomendarse su empleo rutinario en la práctica clínica.

A modo orientativo, puede recurrirse a la utilización de los marcadores de estado nutricional indicados en la Tabla 1 durante las diferentes fases evolutivas de los pacientes críticos. Si bien tampoco existen recomendaciones sobre la frecuencia con la que deben solicitarse los marcadores de seguimiento nutricional, especialmente los bioquímicos, es una práctica muy extendida la de solicitar una determinación analítica «de seguimiento nutricional»con periodicidad semanal durante la estancia de los pacientes en UCI.

DEFINICIÓN DE LAS HERRAMIENTAS PARA EL DIAGNÓSTICO DE LA DESNUTRICIÓN HOSPITALARIA

Es necesario realizar un tamizaje nutricional de todos los pacientes a su ingreso hospitalario y ocasionalmente en el preingreso, para determinar su situación de riesgo nutricional. El tamizaje debe de ser sencillo y aplicable por personal no especializado, y se utilizará para identificar a los pacientes con riesgo nutricional tanto al ingreso como durante su hospitalización. Por ello, todos los pacientes deberán ser reevaluados a lo largo de la evolución. El método de tamizaje elegido debe ser evaluado a nivel de validez predictiva, validez de contenido y variación interobservador. Entre sus características, deben destacar la facilidad de aplicación, la facilidad de comprensión y la aceptabilidad por paciente y profesionales sanitarios. Debe haber relación con el plan de tratamiento y debe ser aplicable a todos los pacientes. El tamizaje debe incluir parámetros clínicos y analíticos. Dentro de los parámetros clínicos deben estar contemplados la edad, el índice de masa corporal, la pérdida de peso en los últimos meses y la ingesta alimentaria. Dentro de los parámetros analíticos se considera recomendable disponer (por orden de prioridad) de albúmina, linfocitos totales y colesterol total. Puede ser de utilidad obtener los datos a través de un 
Tabla 1. M arcadores del estado nutricional en pacientes críticos

\begin{tabular}{|cl|}
\hline Probable utilidad & Parámetros \\
\hline Valoración de malnutrición al ingreso & Pérdida de peso \\
& Indice de masa corporal \\
& Indice creatinina/talla \\
Renutrición & Colesterol sérico \\
& Valoración subjetiva global \\
& 3-metil histidina \\
& Balance nitrogenado \\
& Prealbúmina \\
Respuesta metabólica & Proteína ligada al retinol \\
& Somatomedina \\
& Excreción de urea \\
& 3-metil-histidina \\
Seguimiento nutricional & Proteínas de fase aguda \\
& Urea \\
& Prealbúmina \\
& Proteína ligada al retinol \\
Pronóstico & Somatomedina \\
& Albúmina \\
& Función muscular \\
& Balance nitrogenado \\
& Albúmina \\
\hline
\end{tabular}

sistema informático. El tamizaje debe también contemplar las demandas nutricionales asociadas a la patología del paciente que motiva el ingreso, a su estado nutricional anterior y en el momento del ingreso y a su capacidad para tomar alimentos. Es altamente recomendable que al resultado final del tamizaje se le dé formato de informe de riesgo nutricional para información y conocimiento del clínico responsable del paciente, así como para constancia documental en la historia clínica. El tamizaje debe repetirse periódicamente a lo largo de la evolución hospitalaria del paciente $\mathrm{y}$, si se detecta malnutrición, la globalidad de los resultados debe de ser evaluada por las unidades de nutrición o el equipo de soporte nutricional.

Finalmente, es fundamental educar a todo el personal sanitario en aras de obtener su colaboración. La puesta en marcha de este método evidencia la necesidad de reevaluar la dotación de personal de las unidades de nutrición 0 equipos de nutrición. Se recomienda el empleo de sistemas de tamizaje específicos para la edad pediátrica.
Diagnóstico y grupos relacionados de diagnóstico (GRDs). Los sistemas de información hospitalaria constituyen herramientas fundamentales de gestión clínica. Aunque las directrices europeas marcan una línea de intervención nutricional en la que gestores, clínicos y servicios generales deben estar implicados, la desnutrición no está bien reflejada en los sistemas de información, lo que conlleva su infra-notificación en los servicios hospitalarios (SSHH) actuales. Por otra parte, tampoco el impacto de la intervención nutricional está suficientemente reflejada en los sistemas de información, lo que también implica su infra-notificación en los SSHH actuales. Los gestores y facultativos no expertos en nutrición desconocen los aspectos relacionados con la desnutrición. Existe un gasto oculto y una herramienta de mejora ignorada.

Existen evidencias de que el diagnóstico de desnutrición e intervención nutricional son costeefectivas. Es por ello que sería conveniente elaborar un catálogo de prestaciones nutricionales disponibles en todos los centros hospitalarios, de acuerdo con su nivel asistencial. 
El personal sanitario de las unidades o equipos de nutrición debe estar implicado en la microgestión hospitalaria y, desde ellas, se debe pedir a los clínicos de los servicios finales la inclusión, en el informe de alta, de la información sobre el diagnóstico de desnutrición y los procedimientos de soporte nutricional (nutrición parenteral; nutrición enteral; suplementos; nutrición complementaria; valoración nutricional; asistencia unidad de nutrición...) que ha recibido el enfermo. Para ello se debe solicitar al servicio de documentación la creación de una cartera de servicios para las actuaciones de la unidad o equipo de nutrición. En cualquier caso hay que considerar el valor documental de los informes de riesgo nutricional obtenidos por la unidad o equipo de nutrición, como resultado de la aplicación del tamizaje al ingreso descrito anteriormente; así como de los informes de caso nutricional emitidos al finalizar los tratamientos con soporte nutricional de NP y NE.

Oportunidad de mejora: De acuerdo con las Recomendaciones del Comité de Ministros del

\section{REFERENCIAS}

1. Mourão F, Amado D, Ravasco P, Marqués Vidal P, CAMILO ME. Nutritional risk and status assessment in surgical patients: a challenge amidst plenty. Nutr Hosp 2004; 19: 83-8.

2. Chan S, McCwen KC, Blackburn GL. Nutrition management in the ICU. Chest 1999; 115: 145-88.

3. Jeejeebhoy K. Nutritional assessment. Gastroenterol Clin North Am 1998; 27: 347-69.

4. Fernández Ortega FJ, Ordoñez González FJ, Blessa MALPICA AL Soporte nutricional del paciente en estado critico: ¿A quién, cómo, cuándo? Nutr Hosp 2005; 20(supl 2): 9-12.

5. JimÉnez Jiménez FJ, López Martínez J, SánchezIzQUiERDO RIERA JA. Nutrición artificial en la insuficiencia renal aguda. Nutr Hosp 2005; 20(supl 2): 18-21.

6. JimÉnez JimÉnez FJ, Montejo Alvarez JC, NúÑez Ruiz R. Nutrición artificial en la insuficiencia hepática. Nutr Hosp 2005; 20 (supl 2): 22-4.

7. Garnacho Montero J, García de Lorenzo A, OrDÓÑEZ GonZÁlEZ FJ. Soporte nutricional en la pancreatitis aguda. Nutr Hosp 2005; 20 (supl 2): 25-7.
Consejo de Europa (Resolución ResAP (2003) 3 sobre alimentación y asistencia nutricional en los hospitales; se pueden detectar numerosos campos donde actuar e introducir mejoras en el pronóstico de los pacientes y en la morbimortalidad.

Teniendo en cuenta que la financiación de los hospitales españoles está influida por los datos recogidos en los sistemas de información de los centros, es fundamental sensibilizar a los servicios finales para que mejoren la información sobre el estado nutricional en los datos estadísticos que se recogen. Ello nos lleva a la necesidad de realizar consensos y acuerdos entre las unidades de nutrición o equipos de soporte nutricional y los servicios de documentación.

Para lograr un alto nivel de eficacia y eficiencia, debemos establecer los criterios técnicos de codificación en las definiciones de desnutrición ${ }^{35}$. La Sociedad Española de Nutrición Parenteral y Enteral (SENPE) ha creado un grupo de trabajo sobre desnutrición ${ }^{36}$, entre cuyos objetivos está la validación de las herramientas para el control y evaluación del estado nutritivo.

8. López Martínez J, Planas M, AÑon JM. Nutrición artificial en la insuficiencia respiratoria. Nutr Hosp 2005; 20 (supl 2): 28-30.

9. Grau Carmona T, Bonet A, Fernández F. Nutrición artificial en la insuficiencia intestinal: síndrome de intestino corto. Enfermedad inflamatoria intestinal. Nutr Hosp 2005; 20 (supl 2): 31-3.

10. López Martínez J, Mesejo Arizmendi A, Montejo GoNZÁLEZ JC. Nutrición artificial en la hiperglucemia y diabetes mellitus en pacientes críticos. Nutr Hosp 2005; 20 (supl 2): 34-7.

11. Sánchez Álvarez C, Núñez Ruiz R, Morán García V. Soporte nutricional en el paciente con neoplasia digestiva. Nutr Hosp 2005; 20 (supl 2): 38-40.

12. Sánchez Álvarez C, Núñez Ruiz R, Conejero García R. Soporte nutricional en el paciente con SIDA. Nutr Hosp 2005; 20 (supl 2): 41-3.

13. Grau Carmona T, Rincón Ferrari MD, Gracia Labajo D. Nutrición artificial en el gran quemado. Nutr Hosp 2005; 20 (supl 2): 44-6.

14. García de Lorenzo A, Acosta Escribano J, Bonet SARIS A. Nutrición artificial en el paciente politraumatizado. Nutr Hosp 2005; 20 (supl 2): 47-50. 
15. Ortiz Leyba C, López Martínez J, Blesa Malpica AL. Nutrición artificial en la sepsis. Nutr Hosp 2005; 20 (Supl 2): 51-53.

16. Mesejo Arizmendi A, Ordóñez GonzÁlez J, Ortiz LeYBA C. Nutrición artificial en el trasplante de células precursoras hematopoyéticas (TCPH). Nutr Hosp 2005; 20 (supl 2): 54-6.

17. García de Lorenzo A, Álvarez J, Calvo MV, de UlíbarRi JI, del Río J, Galbán C et al. Conclusiones del II Foro de Debate SENPE sobre desnutrición hospitalaria. Nutr Hosp 2005; 20: 82-7.

18. Acosta Escribano J, Gómez-Telo V, Ruiz Santana S. Valoración del estado nutricional en el paciente grave. Nutr Hosp 2005; 20(supl 2): 5-8.

19. SABol VK. Nutrition assessment of the critically ill adult. AACN Clin Issues 2004; 15: 595-606.

20. Acuña K, Portela M, Costa-Matos A, Bora L, Rosa M, WaITZBerg D, CRUZ T. Nutritional assessment of adult patients admitted to a hospital of the Amazon Region. Nutr Hosp 2003; 18(3): 138-46.

21. Campilo B, Pailaud E, Uzan I, Merder I, Abdeliauu M, PerenNec J et al. Value of body mass index in the detection of severe malnutrition: influence of the pathology and changes in anthropometric parameters. Clin Nutr 2004; 23: 551-9.

22. Pianas Vila M, Pérez-Portabelia C, Virgil Casas N. Valoración del estado nutricional del adulto. En: Gil Hernández A. Tratado de nutrición. Tomo III. Madrid. Acción Médica- SENPE. 2005: 117-148.

23. Kiein S, Kinney J, Jeejeebhoy K, Alpers D, HeuersteIN M, MuRRAY M ET AL. Nutrition support in clinical practice: Review of published data and recommendations for future research directions. Am J Clin Nutr 1997; 66: 683-706.

24. Detsky AS, McLaughuin JR, Baker JP, Johnston N, WhitTAKER S, MeNDelson RA et al. What is subjective global assessment of nutritional status? JPEN J Parenter Enteral Nutr 1987; 11: 8-13.

25. Bistrian BR, Blackburn GL, Sherman M, Scrimshaw NS. Therapeutic index of nutritional depletion in hospitalized patients. Surg Gynecol Obstet 1975; 141: 512-16.

26. Carney DE, Meguid MM. Current concepts in nutritional assessment. Arch Surg 2002; 137: 42-5.
27. Daley BJ, Bristian BR. Nutritional assessment. En: Zaloga GP (ED) Nutrition in critical care. ST. Louis. Mosby 1994: 9-33.

28. Boles JM, Garre MA, Youinou Py, Mialon P, Menez JF, JouQuan J ET al. Nutritional status in intensive care patients: Evaluation in 84 unselected patients. Crit Care Med 1983; 11: 87-90.

29. De Paz R, Fernández Navarro R. Manejo, prevención y control del síndrome anémico secundario a deficiencia férrica. Nutr Hosp 2005; 20(5): 364-67.

30. Muñoz M, Romero A, Morales MN, Campos A, García-Erce J, Ramírez G. Iron metabolism, inflammation and anemia in critically ill patients. A crosssectional study. Nutr Hosp 2005; 20(2): 115-20.

31. Kuzuya M, Kanda S, Koike T, Suzuki Y, Iguchi A. Lack of correlation between total lymphocyte count and nutritional status in the elderly. Clin Nutr 2005; 24: 427-32.

32. Faintuch J, Cabraitz R, Martin Neto aR, Salem M, Yagi O, Leme RB et al. Prognostic interest of cholesterol levels in malnourished patients with esophageal carcinoma. Clin Nutr 1992; 11 (Supp 1): 72.

33. Kyle UG, Bosaeus I, De Lorenzo AD, Deuremberg $P$, ELA M, Gómez JM et aL. Bioelectrical impedance analysis. Part I: review of principles and methods. Clin Nutr 2004; 23: 1226-43.

34. Bewdido Guerrero D, Carreira Arias J, Martínez Olmos M. Análisis de la composición corporal. En: Gil Hernández A. Tratado de nutrición. Tomo III. Madrid. Acción Médica- SENPE. 2005: 149-91.

35. Valero Zanuy MA, Diez Domingo L, El Kadaoui Calvo N, Jiménez Massa AE, Rodríguez Rosas H, LEón SANZ M. ¿Son las herramientas recomendadas por ASPEN y la ESPEN equiparables en la valoración del estado nutricional? Nutr Hosp 2005; 20: 259-67.

36. Ulíbarri JI, González-Madroño A, de Viuar NGP, González P, GonzÁlez B, Mancha A et al. CONUT: una herramienta para controlar el estado nutritivo. Primera validación en una población hospitalaria. Nutr Hosp 2005; 20: 38-45. 